\title{
Successful use of Gasserian ganglion block for maxillo-mandibular fixation in a patient with severe pulmonary dysfunction-a case report
}

\author{
Prasanna Vadhanan ${ }^{1,2}$ \\ 'Department of Anaesthesiology, Vinayaka Missions Medical College, Vinayaka Missions Research foundation, Karaikal, Puducherry, India \\ ${ }^{2}$ Vairam Multispeciality Hospitals, Mayiladuthurai, India
}

\begin{abstract}
Various anesthetic techniques have been utilized for maxillo-mandibular fixation. We report the case of a patient with bilateral condylar and zygomatic arch fractures who had severe pulmonary dysfunction. The patient was administered bilateral image-guided Gasserian ganglion block through the foramen ovale to achieve surgical anesthesia. The technical details, advantages, and disadvantages of this rather unusual technique are discussed. The procedure could be a feasible technique when performed meticulously in cases where other approaches are deemed difficult.
\end{abstract}

Keywords: Gasserian Ganglion; Mandibular Fractures.

This is an Open Access article distributed under the terms of the Creative Commons Attribution Non-Commercial License (http://creativecommons.org/licenses/by-nc/4.0/) which permits unrestricted non-commercial use, distribution, and reproduction in any medium, provided the original work is properly cited.

\section{INTRODUCTION}

Maxillo-mandibular fixation (MMF, intermaxillary fixation), which is performed for the reduction and stabilization of mandibular fractures is a painful procedure that requires appropriate anesthesia. Various anesthetic techniques, including local infiltrations, nerve blocks, application of topical anesthetic gels, and general anesthesia have been employed. The trigeminal (Gasserian) ganglion, which is the largest sensory ganglion, gives rise to the ophthalmic, maxillary, and mandibular nerves that innervate the face. The ganglion is accessible via the foramen ovale, through which the mandibular nerve exits. We report a rather unusual technique, the bilateral Gasserian ganglion block, in an elderly man who had sustained bilateral mandibular condyle and zygomatic fractures. Other anesthetic techniques were considered unsuitable due to the local pathology and comorbid conditions of the patient.

\section{CASE REPORT}

A 78-year-old man presented with bilateral condylar and zygomatic arch fractures following a road traffic accident. Clinical examination revealed that the patient had irregular dentition with several missing teeth and restricted mouth opening (1 finger breadth) due to the fracture. He had a history of chronic obstructive pulmonary disease with severe functional limitations, punctuated by frequent exacerbations. His modified

Received: June 30, 2020 - Revised: August 13, 2020 - Accepted: August 19, 2020

Corresponding Author: Prasanna Vadhanan, Dip. Pain Management. Department of Anaesthesiology, Vinayaka Missions Medical College, Vinayaka Missions Research foundation, Karaikal, Puducherry, India, Consultant Anesthesiologist, Vairam Multispeciality Hospitals, Mayiladuthurai, India

Phone: +919486489690 E-mail: vadhanan.prasanna@gmail.com, prasanna12778@vmmckkl.edu.in

Copyright(c) 2020 Journal of Dental Anesthesia and Pain Medicine 
Medical Research Council Score, used to assess the degree of disability, was 3 (stops for breath after walking for 100 meters or after few minutes on the level). The last exacerbation occurred before 4 weeks, during which the patient was hospitalized for 3 days. He was on irregular treatment and had stopped his medications since discharge. Pulmonary function tests performed during the previous hospitalization showed a FEV1/FVC of $60 \%$ and FEV1 of 35\% (FEV1-0.99 L, FVC -1.64 L) indicating severe disease (GOLD stage III). During admission, the patient had persistent cough with considerable sputum, bilateral wheeze, and diminished air entry on the right side. His chest radiograph showed fibrosis of the right upper lobe and bronchiectatic changes. He was treated with antibiotics, inhalational bronchodilators, and steroids. Despite optimization, the patient demonstrated marginal clinical improvement. Wheezing and cough improved, and he was able to lie down in the supine position without any discomfort. His room air oxygen saturation was $96 \%$. Heart rate and blood pressure were within normal limits (88/minute and 150/80 mm Hg, respectively). Results of routine blood investigations (hemoglobin, total white blood cell and differential counts, blood sugar, urea, creatinine) were normal. Electrocardiogram showed a right axis deviation with occasional atrial ectopics.

Monitored anesthetic care was the technique of choice considering the high risk associated with general anesthesia. Midazolam $1 \mathrm{mg}$ and glycopyrrolate $0.2 \mathrm{mg}$ were administered intravenously as premedication. Routine monitoring was initiated with three-lead electrocardiogram, pulse oximetry, and noninvasive blood pressure assessments. Inferior alveolar nerve block was administered by the closed mouth technique with $3 \mathrm{ml}$ of buffered 2\% lignocaine (Neon Laboratories, India) on either side by the surgeon. Despite this, the patient's mouth could not be opened adequately to allow surgical access and he experienced considerable pain. Further attempts with local infiltrations were not tolerated by the patient, and he became agitated. A decision to block the maxillary and mandibular nerves more proximally through the foramen ovale under fluoroscopic guidance was taken, as the local anatomy precluded other approaches. Moreover, the author, an anesthesiologist, regularly performs trigeminal ganglion radiofrequency ablations for management of trigeminal neuralgia. After placing the patient in the supine position, the head was extended and fixed with adhesive tape. A $20^{\circ}$ caudal tilt of the image intensifier along with an ipsilateral $45^{\circ}$ tilt brought the foramen ovale into view (Fig. 1A). After skin infiltration, a $23 \mathrm{G}$ spinal needle with a bent tip was introduced using an end on view to touch the lateral margin of the foramen ovale. The needle was slipped into the foramen at which point the patient complained of paresthesia. A lateral image was obtained, which showed the needle passing through the foramen across the petrous part of the temporal bone (Fig. 1B). After negative aspiration for blood and cerebrospinal fluid, $3 \mathrm{ml}$ of $0.5 \%$ bupivacaine was slowly injected and the procedure was repeated on the other side. The patient reported immediate pain relief, and after five minutes, complete anesthesia of the mandibular and maxillary nerves was achieved. Full mouth opening was possible (Fig. 1C) and MMF was performed with arch bars and wires. Conservative management of the zygomatic fracture was planned. The surgery was performed in the $15^{\circ}$ head-up position and continuous suctioning was ensured to prevent any blood or secretions from reaching the hypopharynx. The duration of the procedure was 45 minutes, during which the patient remained conscious and recovered uneventfully.

\section{DISCUSSION}

General anesthesia is the preferred technique for complex faciomaxillary fractures because it results in adequate muscle relaxation, thus facilitating mouth opening and fracture reduction [1]. It is also the technique of choice in uncooperative patients and in those at extremes of age. A secured airway offers protection against aspiration of blood and secretions. However, this 


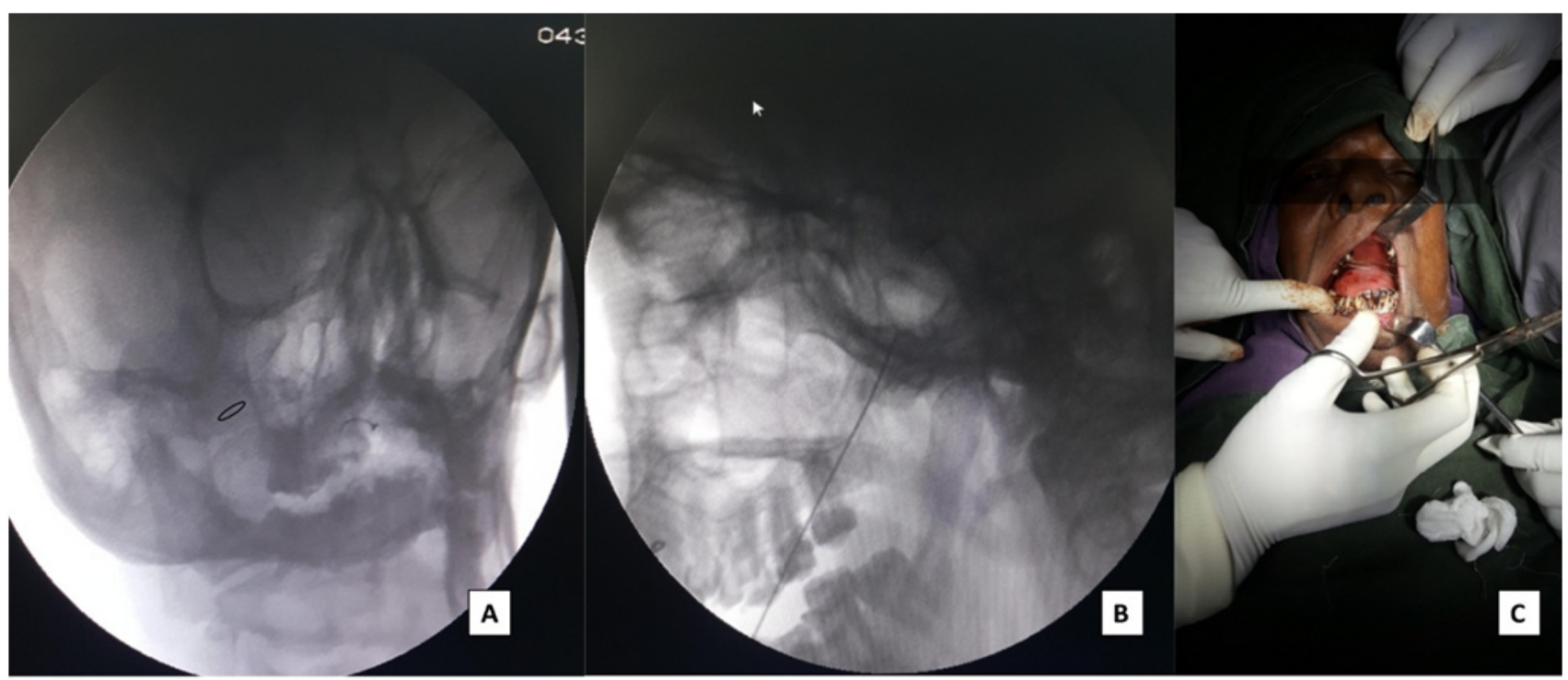

Fig. 1. (A) Caudal and ipsilateral tilt showing position of the foramen ovale between the posterior wall of the maxillary sinus and the mandible. (B) Lateral view showing the needle trajectory passing through the foramen ovale and petrous part of the temporal bone. (C) Intraoperative image demonstrating adequate mouth opening.

procedure can be performed under nerve blocks, local infiltrations, or topical anesthetic gels [2]. Our patient did not tolerate local infiltrations and his mouth opening was severely restricted. The condition of his lungs posed significant risk for adverse perioperative events such as bronchospasm, atelectasis, hypoxia, hypercarbia, and the possibility of postoperative mechanical ventilation.

Combined maxillary and mandibular nerve blocks using an extraoral approach may be effective [3]; however, the condylar and zygomatic fractures rendered this approach difficult. Identification of the coronoid notch and pterygoid plate, which is the key step in extraoral maxillary and mandibular nerve blocks, is difficult in patients with such fractures. Distorted anatomy might lead to a higher incidence of vascular punctures and failed blocks. The success rates of such blind blocks are lower; however, nerve stimulator guidance might be helpful [4]. In contrast, the foramen ovale is a reliably visualized structure that can be targeted accurately. The mandibular condyles receive innervation from the auriculotemporal nerve [5], which takes off proximally from the posterior trunk of the mandibular nerve, before the latter divides into inferior alveolar and lingual nerves. Distal blocks (such as the inferior alveolar nerve block) may not result in effective analgesia of the condylar area, and is associated with a failure rate of $15-20 \%$ [6]. Moreover, anatomical variations of the auriculotemporal [7] and inferior alveolar nerves might result in incomplete or failed nerve blocks [8]. A more proximal approach through the foramen ovale essentially avoids this problem. Maxillary nerve block is also required for complete surgical anesthesia prior to MMF, which can be achieved by a single injection through the foramen ovale.

The Gasserian ganglion is the largest sensory ganglion and is akin to the dorsal root ganglion of the spinal nerve. It lies in a dural invagination (Meckel's cave) in the middle cranial fossa near the apex of the petrous part of the temporal bone, lateral to the internal carotid artery and cavernous sinus. The three branches of the trigeminal nerve arise from the anterior portion of the ganglion and exit the skull through the superior orbital fissure (ophthalmic), foramen rotundum (maxillary), and foramen ovale (mandibular). The ganglion lies superior and posterior to the foramen ovale and can be approached through this foramen.

Gasserian ganglion block through the foramen ovale is usually performed as a diagnostic/therapeutic procedure 
for various painful conditions that affect the trigeminal nerve distribution, and can be used as an anesthetic technique for facial fractures. To the best of our knowledge, to date, the anesthetic application of this technique has not been reported. During diagnostic Gasserian ganglion block, which is occasionally performed before a more definitive procedure such as radiofrequency ablation, position of the needle and absence of intravascular placement are confirmed by injecting a radiopaque dye. This was not done in our patient as the procedure was bilateral, and injection of the dye on one side could render visualization of the second needle tip difficult in lateral projections. Presence of the dye could also interfere with spread of the local anesthetic in small, confined areas. Bupivacaine 0.5\% was preferred over lignocaine due to its longer duration of action, in spite of its relative cardiotoxicity. Careful aspiration of blood or cerebrospinal fluid was performed and the injection was administered in $0.2 \mathrm{ml}$ increments under constant monitoring.

The chief limitation of this approach lies in its technically demanding nature. Considerable experience is required to locate the foramen ovale and navigate the needle to the target under radiographic guidance. The degree of caudal and ipsilateral $\mathrm{C}$-arm tilt required to visualize the foramen ovale is variable and might not be visualized completely in certain cases [9]. We used a bent-tip needle, which enables easy manipulation and is commonly used in various image-guided interventional pain procedures, as it aids in avoiding multiple needle passes.

Some possible complications of this technique are as follows. Intravascular (arterial or venous) injection of local anesthetics is a dreaded complication; therefore, careful aspiration and injection of drugs in small boluses and continuous monitoring is recommended. The volume of drug injected is empirical and excess volumes might block the V1 (ophthalmic) branch of the fifth cranial nerve, resulting in corneal anesthesia necessitating eye protection until the block wears off. During normal course, the needle can potentially damage various structures, including the facial vein and artery, Stensen duct, internal maxillary artery, and pterygoid venous plexus. Operator experience significantly influences the incidence of these complications and most of them are transient and self-limiting. Deep insertion of the needle might cause dural puncture and cerebrospinal fluid leak (the posterior part of the ganglion is bathed in cerebrospinal fluid), brain injury (temporal lobe of the brain is superior to the ganglion), and infection. On the other hand, a medial needle trajectory can injure the carotid artery in the cavernous sinus. Hence, it is recommended to always aim for the lateral margin of the foramen (for mandibular nerve block) and continuously assess the depth of insertion by lateral fluoroscopy. The addition of a nerve stimulator and performing sensory stimulation might be helpful in determining the final position of the needle. A detailed review of measures to avoid or minimize these complications, ideal needle trajectories, and depth has been published by Peris-Celda et al. [9].

In conclusion, we have reported the utilization of combined maxillary and mandibular nerve blocks near their origins from the Gasserian ganglion through the foramen ovale for fixation of mandibular fractures. This technique, when performed by experienced operators, provides adequate surgical analgesia for faciomaxillary surgeries. The advantages of this procedure are a single injection, clear visualization of the target, need for smaller volumes of the local anesthetic, and a dense blockade. However, caution should be exercised, as this procedure has the potential to cause several serious complications.

\section{AUHHOR ORCIDS}

Prasanna Vadhanan: https://orcid.org/0000-0001-6060-3880

\section{AUHOR BONIRBUIONS}

Prasanna Vadhanan: Writing, review, \& editing

ACKNOWLEDGEMENTS: The author wishes to acknowledge Dr. Balaji Mahalingam, MDS (Consultant Oral and 
Maxillofacial Surgeon, Kauvery Hospitals, Trichy, India), Dr. Rajasimman, BDS (Consultant Dental Surgeon, India), Dr. Rajasekaran R (Consultant, Orthopedics and Trauma Surgery, Vairam Hospitals, Mayiladuthurai, India), and Dr. Narendren Ganesh, MD (Assistant Professor, Department of Anaesthesiology, Vinayaka Missions Medical College, Karaikal, India) for their contributions.

Written informed consent was obtained from the patient for publication of this manuscript. The Institutional Ethics Committee of Vinayaka Missions Medical College, Karaikal, India approved this manuscript.

CONFLICT OF INTEREST: The author declared no competing interests.

\section{REFERENCES}

1. Stein KM, Titler S. Maxillomandibular fixation and anesthesia management. AANA J 2017; 85: 469-77.

2. Jeong YJ, Kim HJ, Kwon H, Shim HS, Seo BF, Jung $\mathrm{SN}$. The use of topical lidocaine gel during intermaxillary fixation procedure. J Craniofac Surg 2016; 27: 475-7.
3. Parthasarathy S, Sripriya R. Fixation of bilateral condylar fractures with maxillary and mandibular nerve blocks. Anesth Essays Res 2015; 9: 281-3.

4. Parate LH, Tejesh CA, Geetha CR, Mohan CVR. Peripheral nerve stimulator-guided mandibular nerve block: a report of three cases. Saudi J Anaesth 2016; 10: 491-2.

5. Davidson JA, Metzinger SE, Tufaro AP, Dellon AL. Clinical implications of the innervation of the temporomandibular joint. J Craniofac Surg 2003; 14: 235-9.

6. Lee CR, Yang HJ. Alternative techniques for failure of conventional inferior alveolar nerve block. J Dent Anesth Pain Med 2019; 19: 125-34.

7. Gülekon N, Anil A, Poyraz A, Peker T, Turgut HB, Karaköse M. Variations in the anatomy of the auriculotemporal nerve. Clin Anat 2005; 18: 15-22.

8. Madan GA, Madan SG, Madan AD. Failure of inferior alveolar nerve block: exploring the alternatives. J Am Dent Assoc 2002; 133: 843-6.

9. Peris-Celda M, Graziano F, Russo V, Mericle RA, Ulm AJ. Foramen ovale puncture, lesioning accuracy, and avoiding complications: microsurgical anatomy study with clinical implications. J Neurosurg 2013; 119: 1176-93. 